\title{
A Spectral Representation for Bounded Non-Selfadjoint Operators
}

\author{
Alexandra Cojuhari · Jerzy Stochel
}

Received: 5 March 2011 / Accepted: 23 September 2011 / Published online: 20 October 2011

(C) The Author(s) 2011. This article is published with open access at Springerlink.com

\begin{abstract}
An integral representation for an arbitrary bounded operator $T$ defined on a Hilbert space $\mathcal{H}$ is given. The representing measure is in general defined on a Jordan curve surrounding the spectrum of $T$. It is obtained as a limit, in a certain weak sense, of a family $\left(F_{r}\right)$ of absolutely continuous measures the Radon-Nikodym derivative of which (with respect to the standard Lebesgue measure on the considered Jordan curve) are described explicitly in terms of the operator $T$ and its adjoint $T^{*}$.
\end{abstract}

Keywords Operator-valued measures and integration - Integral representation * Functional calculus $\cdot$ Spectral measure

Mathematics Subject Classification (2000) $\quad 46 \mathrm{G} 10 \cdot 47 \mathrm{~A} 60 \cdot 47 \mathrm{~A} 25$

\section{Introduction}

In this paper we give integral representations for bounded operators defined on Hilbert spaces. Specifically, for an arbitrary bounded operator $T$ on a Hilbert space $\mathcal{H}$, we propose a construction of an operator-valued measure $F$ representing $T$. The measure

Communicated by Guest Editors L. Littlejohn and J. Stochel.

\footnotetext{
A. Cojuhari $(\varangle)$

Institute of Mathematics, Jagiellonian University, ul. Łojasiewicza 6, 30348 Cracow, Poland e-mail: aliuncic@yahoo.com; Alexandra.Cojuhari@im.uj.edu.pl

J. Stochel

Department of Applied Mathematics, AGH University of Science and Technology,

Al. Mickiewicza 30, 30-059 Cracow, Poland

e-mail: stochel@uci.agh.edu.pl
} 
$F$ is defined on the $\sigma$-field of Borel sets in the boundary of a Jordan domain containing the spectrum of the operator $T$.

The problems concerning spectral representations of general non-selfadjoint operators were always taken into consideration by many authors, and to this point there is a vast number of publications in this area. We refer to the volumes $[3,4]$, especially $[5,15]$ for a comprehensive representation of the theory and discussions on perspectives for applications. An important approach to develop an adequate spectral analysis for non-normal operators is based on the dilation theory of constructions initiated by Sz-Nagy and letter developed in collaboration with Foiaş (see [14] and the literature cited there). Another aspect of the theory is outlined by the methods used in [6,7]. In particular, in [6,7] integral representations for bounded operators are obtained via the concept of spectral sets introduced by von Neumann [16]. Note also the authors's work [1] for some related results for operators considered on Banach spaces.

In this paper we proceed from a family of operator-valued measures $\left(F_{r}\right)(0<r<1)$ defined on the Borel sets of the unit circle of the complex plane by means of an operator-valued function (a kind of Poisson kernel) $\Phi(z ; T)$ (cf. (2.1)). The desired measure $F$ representing the operator $T$ is obtained as a weak (in fact in a weak*-topology) limit of $F_{r}$. The representing measure $F$ determines a functional calculus, consistent with the one developed by Sz-Nagy and Foiaş [14] and also by Schreiber [12]. It should be mentioned that the resulting functional calculus in general is not multiplicative, but from the proposed construction it is easy to describe restrictions on functions in order to preserve the multiplicative property as well. In particular, as a consequence, it follows that the measure $F$ represents a spectral measure if and only if the operator $T$ is unitary on $\mathcal{H}$.

The paper is organized as follows. In Sect. 2 we give spectral representation for linear contractions on Hilbert spaces. The general case of a Jordan curve is considered in Sect. 3.

\section{Representing Measures}

Let $\mathcal{H}$ be a complex Hilbert space. The inner product and the norm are denoted by $\langle\cdot, \cdot\rangle$ and $\|\cdot\|$, respectively. We denote by $\mathcal{B}(\mathcal{H})$ the set of all bounded linear operators on $\mathcal{H}$. Consider an operator $T \in \mathcal{B}(\mathcal{H})$ and define for all complex numbers $z$ such that $\|z T\|<1$ the following operator-valued function

$$
\Phi(z ; T)=\sum_{n=-\infty}^{+\infty}(\bar{z} T)^{n},
$$

where $\bar{z}$ is the complex conjugate of $z, T^{*}$ denotes the adjoint of $T$, and with the agreement that $(\bar{z} T)^{0}=I$ and $(\bar{z} T)^{n}=\left((\bar{z} T)^{*}\right)^{-n}$ for $n=-1,-2, \ldots$

The operator-valued function $\Phi(z ; T)$ can be also represented as follows

$$
\begin{aligned}
\Phi(z ; T) & =(I-\bar{z} T)^{-1}+z T^{*}\left(I-z T^{*}\right)^{-1} \\
& =\left(I-z T^{*}\right)^{-1}\left[I-z T^{*}+z T^{*}(I-\bar{z} T)\right](I-\bar{z} T)^{-1} \\
& =\left(I-z T^{*}\right)^{-1}\left(I-|z|^{2} T^{*} T\right)(I-\bar{z} T)^{-1},
\end{aligned}
$$


i.e.

$$
\Phi(z ; T)=\left(I-z T^{*}\right)^{-1}\left(I-|z|^{2} T^{*} T\right)(I-\bar{z} T)^{-1}, \quad\|z T\|<1 .
$$

We see that, for a fixed $z$ such that $\|z T\|<1, \Phi(z ; T)$ is a positive operator (bounded) on $\mathcal{H}$ in the sense of quadratic forms, i.e.

$$
1^{0} . \Phi(z ; T) \geq 0, \quad\|z T\|<1,
$$

and, that

$$
2^{0} . \quad \frac{1}{2 \pi i} \int_{\mathbb{T}_{r}} \Phi(z ; T) \frac{d z}{z}=I,
$$

where $r$ is any positive real number such that $\|r T\|<1 ; \mathbb{T}_{r}$ denotes the circle $|z|=r$ oriented in the positive sense.

Next, we suppose that $T$ is a contraction on $\mathcal{H}$, with hence $\|T\| \leq 1$ (as will be seen there is no loss of generality), and let

$$
F_{r}(\delta)=\int_{\delta} \Phi(r z ; T) d \tau
$$

for $r<1$ and for any Borel set $\delta$ in the unit circle $\mathbb{T}$ (we write simply $\mathbb{T}$ instead of $\mathbb{T}_{1}$ ); $\tau$ denotes the normalized standard Lebesgue measure on $\mathbb{T}$.

For a fixed $r, 0<r<1$, the $\mathcal{B}(\mathcal{H})$-valued set function $F_{r}$, defined on the $\sigma$-field of Borel sets in $\mathbb{T}$, is strongly countable additive. The properties $1^{0}$ and $2^{0}$ provide $F_{r}$ to be a semi-spectral measure (for the concept of a semi-spectral measure see, for instance [10]). Obviously, $F_{r}$ is bounded in the sense that the norms of the operators in its range are bounded.

Having the measures $F_{r}(0<r<1)$ we can integrate scalar measurable functions on $\mathbb{T}$. It can be started with simple functions, and then going over to the general case of bounded functions from $L_{\infty}(\mathbb{T}, \tau)$. For a function $f \in L_{\infty}(\mathbb{T}, \tau)$ we write

$$
f_{r}(T)=\int_{\mathbb{T}} f(z) d F_{r},
$$

or, which is one and the same, since $d F_{r}=\Phi_{r}(z ; T) d \tau$ (cf. (2.3))

$$
f_{r}(T)=\int_{\mathbb{T}} f(z) \Phi(r z ; T) d \tau .
$$

In particular, for complex-valued functions $f$ with absolutely convergent Fourier series (the Wiener class $W$ ), that is for functions $f$ which can be represented in the form 


$$
f(z)=\sum_{n=-\infty}^{\infty} c_{n} z^{n}, \quad z \in \mathbb{T}
$$

with

$$
\sum_{n=-\infty}^{\infty}\left|c_{n}\right|<\infty
$$

the integration over $\mathbb{T}$ with respect to $F_{r}$ has sense and, as is easy to calculate,

$$
f_{r}(T)=\sum_{n=-\infty}^{\infty} c_{n} r^{|n|} T^{n} \quad(0<r<1) .
$$

We point out that the map $f \rightarrow f_{r}(T)$ of $L_{\infty}(\mathbb{T}, \tau)$ into $\mathcal{B}(\mathcal{H})$ given by (2.5) is a linear operator (it will be shown below that it is bounded). In general it is not multiplicative (cf. (2.8), below), but, since the operator-valued measure $F_{r}$ is positive, the involution is preserved, namely there holds

$$
f_{r}(T)^{*}=\int_{\mathbb{T}} \overline{f(z)} \Phi(r z ; T) d \tau,
$$

for each $f \in L_{\infty}(\mathbb{T}, \tau)$ and $0<r<1$.

Concerning multiplicativity, the following formula is clear by property $2^{0}$ and relation $(2.5)$

$$
\begin{aligned}
f_{r}(T) g_{r}(T)= & \int_{\mathbb{T}} f(z) g(z) \Phi(r z ; T) d \tau \\
& -\int_{\mathbb{T}}\left(f(z) I-f_{r}(T)\right) \Phi(r z ; T)\left(g(z) I-g_{r}(T)\right) d \tau
\end{aligned}
$$

for every $f, g \in L_{\infty}(\mathbb{T}, \tau)$ and $0<r<1$.

Taking $g=\bar{f}$ in (2.8) we obtain

$$
f_{r}(T) f_{r}(T)^{*} \leq \int_{\mathbb{T}}|f(z)|^{2} \Phi(r z ; T) d \tau .
$$

Therefore

$$
\begin{aligned}
\left\|f_{r}(T) x\right\|^{2} & \leq \int_{\mathbb{T}}|f(z)|^{2}\langle\Phi(r z ; T) x, x\rangle d \tau \\
& \leq\|f\|_{\infty}^{2} \int_{\mathbb{T}}\langle\Phi(r z ; T) x, x\rangle d \tau=\|f\|_{\infty}^{2}\|x\|^{2}
\end{aligned}
$$

i.e.

$$
\left\|f_{r}(T) x\right\| \leq\|f\|_{\infty}^{2}\|x\|, \quad 0<r<1,
$$

for each $f \in L_{\infty}(\mathbb{T}, \tau)$ and $x \in \mathcal{H}$. 
Thus

$$
\left\|f_{r}(T)\right\| \leq\|f\|_{\infty}^{2}, \quad f \in L_{\infty}(\mathbb{T}, \tau)
$$

that means, in particular, that the map $f \rightarrow f_{r}(T)$ defines a bounded operator from $L_{\infty}(\mathbb{T}, \tau)$ into $\mathcal{B}(\mathcal{H})$.

Next, denote by $\mathcal{P}$ the set of all trigonometric polynomials of the form

$$
p(z)=\sum_{k=-m}^{m} c_{k} z^{k}, \quad z \in \mathbb{T},
$$

with coefficients $c_{k} \in \mathbb{C}(k=0, \pm 1, \ldots, \pm m)$. Pick $p \in \mathcal{P}$ and observe that $p_{r}(T)$ which can be represented as (cf. (2.6))

$$
p_{r}(T)=\sum_{k=-m}^{m} c_{k} r^{|k|} T^{k}
$$

converges in the norm topology of $\mathcal{B}(\mathcal{H})$ to

$$
p(T)=\sum_{k=-m}^{m} c_{k} T^{k}
$$

as $r \rightarrow 1$.

Therefore, it can be defined an operator $\mathcal{K}: \mathcal{P} \rightarrow \mathcal{B}(\mathcal{H})$ by

$$
\mathcal{K} p=u-\lim _{r \rightarrow 1-0} \int_{\mathbb{T}} p(z) \Phi(r z ; T) d \tau, \quad p \in \mathcal{P} .
$$

Obviously, the operator $\mathcal{K}$ is linear. It is also bounded because of (2.10). Hence, since $\mathcal{P}$ is a dense set in $C(\mathbb{T})$, the operator $\mathcal{K}$ can be extended by continuity to $C(\mathbb{T})$. For the obtained extension we preserve the same notation $\mathcal{K}$. Due to the Riesz Representation Theorem for operators defined on the spaces of continuous functions (see [2, Theorem VI. 2.1, pag. 152]) there exists a uniquely determined operator-valued measure $F$ defined on the Borel sets in $\mathbb{T}$ such that the associated scalar measures $\mu_{x, y}^{F}$ of $F$, i.e. the complex-valued measures defined by $\mu_{x, y}^{F}(\delta)=\langle F(\delta) x, y\rangle$ for any Borel set $\delta$ in $\mathbb{T}$ and $x, y \in \mathcal{H}$ are regular on $\mathbb{T}$ (we agree then to term $F$ regular on $\mathbb{T}$ ) and for which

$$
\mathcal{K} f=\int_{\mathbb{T}} f d F, \quad f \in C(\mathbb{T})
$$

Moreover,

$$
\|F\|=\|\mathcal{K}\|
$$


We define the norm $\|F\|$ as the supremum of the quantities $\left\|\sum_{1}^{n} \lambda_{k} F\left(\delta_{k}\right)\right\|$, where $\delta_{k}(k=1, \ldots, n)$ is a finite sequence of mutually disjoint Borel sets in $\mathbb{T}$ and $\lambda_{k}$ being complex numbers with $\left|\lambda_{k}\right| \leq 1(k=1, \ldots, n)$. In view of (2.13) the operator-valued measure $F$ is bounded in $\mathbb{T}$ (cf. [2, Proposition I. 1.1., pag. 4]).

In addition, we point out that the operator-valued measure $F$ is positive, i.e.

$$
\mu_{x, x}^{F}(\delta)=\langle F(\delta) x, x\rangle \geq 0
$$

for any Borel set $\delta$ in $\mathbb{T}$ and $x \in \mathcal{H}$. This fact follows easily from the integral representation (2.12) by using relation (2.13) and $\mathcal{K} 1=I$.

The following summarizes the above discussion.

Theorem 2.1 Let $T$ be a contraction on a Hilbert space $\mathcal{H}$ and let $\Phi(z ; T)$ be the operator-valued function defined by (2.1). Then

(i) The operator-valued function $\Phi(z ; T)$ determines uniquely a $\mathcal{B}(\mathcal{H})$-valued measure $F$ defined on the Borel sets in $\mathbb{T}$ such that

$$
\int_{\mathbb{T}} p(z) d F=u-\lim _{r \rightarrow 1-0} \int_{\mathbb{T}} p(z) \Phi(r z ; T) d \tau
$$

for all trigonometric polynomials $p \in \mathcal{P}$;

(ii) The operator-valued measure $F$ is bounded, positive and regular on $\mathbb{T}$;

(iii) There holds the following integral representation

$$
T=\int_{\mathbb{T}} z d F
$$

Moreover,

(iv) The measure $F$ represents the powers of $T$ by

$$
T^{n}=\int_{\mathbb{T}} z^{n} d F, \quad n=0, \pm 1, \ldots,
$$

where (by above agreement) $T^{n}=\left(T^{*}\right)^{-n}$ for $n=-1,-2, \ldots$

Remark 2.2 Theorem 2.1 can be reformulated for an arbitrary bounded operator $T$ on $\mathcal{H}$ in an obvious way considering $\mathbb{T}_{r}$ with $r=\|T\|$ instead of $\mathbb{T}$.

According to the assertion (i) in Theorem 2.1 the measures $F_{r}$ converge to $F$ in a weak sense. $F_{r}(\delta)$ for fixed $\delta$ (but arbitrary) does not in general converge to $F(\delta)$ in one operator topology or another. This can be explained as follows. Each of $F_{r}$ is absolutely continuous with respect to the Lebesque measure $\tau$ and if, for instance, $F_{r}$ (or a sequence $\left(F_{r_{n}}\right)$ ) converges weakly on Borel sets $\delta$ to $F(\delta)$, then due to of Vitali-Hahn-Saks-Nikodym Theorem (see for instance, [2, Theorem I.4.8 and 
Corollary I.5.6, pag. 23-24]) $F$ must be also absolutely continuous with respect to $\tau$. But the latter is not true if $T$ is, respectively, not an absolutely continuous operator. Concerning the concept of absolute continuity of contractions we cite the works $[12,13]$ (see also the references cited there).

In case that the spectral radius $r(T)$ of $T$ is strongly less than the norm of $T$ the following is true however.

Theorem 2.3 Let $T$ be a contraction on $\mathcal{H}$ supposing that $r(T)<1$. Then the operator-valued measure defined by (2.14) in Theorem 2.1 is determined by

$$
F(\delta)=\int_{\delta} \Phi(z ; T) d \tau
$$

for each Borel set in $\mathbb{T}$ and, therefore, it is absolutely continuous with respect to $\tau$.

Proof It is clear that $\mathbb{T}$ is contained in the resolvent set of the operator $T$ and $\Phi(r z ; T)$ converges in the norm topology of $\mathcal{B}(\mathcal{H})$ to $\Phi(z ; T)$ as $r \rightarrow 1-0$.

Remark 2.4 The operator-valued function $\Phi(z ; T), z \in \mathbb{T}$, in (2.17) can be represented in the form

$$
\Phi(z ; T)=\left(D_{T} R(z ; T)\right)^{*} D_{T} R(z ; T), \quad z \in \mathbb{T},
$$

where $\Phi(z ; T)=(z I-T)^{-1}$ denotes the resolvent and $D_{T}$ the defect operator $D_{T}=\left(I-T^{*} T\right)^{1 / 2}$ of the operator $T$.

The result given by Theorem 2.3 is closely connected to results of Lebow [8] and Mlak [9] where conditions under which a contraction has spectral radius less than one were given. In particular, in [9] was shown that if for every $x, y \in \mathcal{H}$ the function $u_{x, y}$ given by

$$
u_{x, y}(z)=\left\langle(I-z T)^{-1} x, y\right\rangle, \quad|z|<1
$$

belongs to the Hardy class $H_{1}$, then $r(T)<1$.

The representing measure $F$ defined by (2.14) determines a functional calculus for $T$. Namely, in connection with (2.16) the operators obtained by integration of $F$-bounded measurable functions on $\mathbb{T}$ with respect to the measure $F$ can be naturally interpreted as functions of $T$. It is seen that this functional calculus in general is not multiplicative. However (cf. (2.5), (2.6) and (2.14)) the multiplicative property is preserved for holomorphic functions and continuous up to the boundary in the domains $|z|<1$ and $|z|>1$, respectively. The corresponding homomorphisms can be extended by Bartle's Bounded Convergence Theorem (see [2, Theorem II. 4.1, pag. 56]) to functions which are limits of uniformly bounded sequences of those already mentioned holomorphic functions, respectively. It should be mentioned that the resulting functional calculus is consistent with that developed by Sz-Nagy and Foiaş [14] in a series of their publications and explained in detail (see also the references cited there). The latter can be explained by the fact that the results are the same in both cases at least 
for functions of the class $\mathcal{H}_{\infty}^{T}$ (see [14, Chapter III, § 2]). For any $f \in \mathcal{H}_{T}^{\infty}$ it can be defined

$$
f(T)=s-\lim _{r \rightarrow 1-0} f(r T),
$$

that correspond to (2.11) and (2.14). Recall that the class $\mathcal{H}_{\infty}^{T}$ is defined as the set of all functions $f \in \mathcal{H}_{\infty}\left(\mathcal{H}_{\infty}\right.$ denotes for the Hardy space) for which the strong limit in (2.17) exists. On the other hand if $U$ denotes the minimal dilation of the contraction operator $T$, then, according to the Sz Nagy-Foiaş functional calculus (cf. [14, Theorems III. 2.1 and III. 2.3]) for $f(T)$ defined by (2.17) there holds

$$
f(T)=\operatorname{prf}(U),
$$

$f(U)$ being calculated by means of the spectral measure of $U$.

Finally, note that in case $T$ is a unitary operator on $\mathcal{H}$ the map $\mathcal{K}$ defined as in the proof of Theorem 2.1 is in addition, multiplicative, i.e. it is an algebraic homomorphism of $C(\mathbb{T})$ into $\mathcal{B}(\mathcal{H})$. Using this fact by standard arguments it can be derived that the values of the measure $F$ are projections in $\mathcal{H}$. Besides they are orthogonal provided that the measure $F$ is positive. Consequently, in this case, $F$ represents a spectral measure in $\mathcal{H}$. Conversely, it is well known that the integration with respect to a spectral measure has the property of multiplicativity. Therefore, if $F$ is a spectral measure, then, due to (2.16), the operator $T$ is unitary on $\mathcal{H}$.

We restrain the above discussions in the following.

Corollary 2.5 Under the hypotheses of Theorem $2.1 F$ is a spectral measure if and only if $T$ is a unitary operator in $\mathcal{H}$.

\section{A General Setting}

The discussions made in the previous section can be carried over to the more general case of an arbitrary bounded operator $T$ which spectrum is surrounded by a Jordan curve $\Gamma$. Denote by $\Omega$ the closed domain with $\partial \Omega=\Gamma$. The classical Riemann's theorem ensures the existence of a conformal mapping $\varphi$ of the interior $\Omega$ on the open unit disk $\mathbb{D}$, but in case the boundary $\partial \Omega$ is a Jordan curve, the map $\varphi$ can be extended continuously on $\Omega$ so that $\mathbb{T}$ corresponds (continuously and one-to-one) to $\partial \Omega(=\Gamma)$. We denote the extension of $\varphi$ on $\Omega$ again by $\varphi$. By the spectral mapping theorem the spectrum of the operator $\varphi(T)$ is, respectively, contained in the closed disk $\overline{\mathbb{D}}$ and, working with $\varphi(T)$ instead of $T$, we can apply the arguments used in the previous section in order to obtain the desired results. However, we can make a direct reference to formula (2.15) for $\varphi(T)$. To this end, we assume that

$$
\|\varphi(T)\| \leq 1
$$

or, equivalently,

$$
I-\varphi(T)^{*} \varphi(T) \geq 0
$$


Then, Theorem 2.1 can be applied, and therefore one can indicate an operator-valued measure $F$ representing $\varphi(T)$ by

$$
\varphi(T)=\int_{\mathbb{T}} z d F .
$$

Next, consider the operator-valued measure $G$ defined on the Borel sets $\partial$ in $\Gamma(=\partial \Omega)$ by

$$
G(\partial)=F(\varphi(\partial)) .
$$

It is seen that the measure $G$ is bounded, positive and regular on $\Gamma$. The change of variable $z=\varphi(\zeta), \zeta \in \Gamma$, in (3.1) leads to the required integral representation for $T$. Namely,

$$
T=\int_{\Gamma} \zeta d G
$$

Before formulating the obtained result we note that, by von Neumann's theory on spectral sets [16], a condition (3.1) is equivalent with the fact that the unit disk $\overline{\mathbb{D}}$ is a spectral set for $T$. But, due to the invariant principle for spectral sets [16] (see also [15]), $\overline{\mathbb{D}}$ is a spectral set for $\varphi(T)$ if and only if $\Omega$ is a spectral set for $T$. Recall that $\Omega$ is a spectral set for the operator $T$ if $\|f(T)\| \leq \max _{\zeta \in \Omega}|f(\zeta)|$ holds true for any analytic function $f$ on $\Omega$.

Theorem 3.1 Let $T$ be a bounded operator on a Hilbert space $\mathcal{H}$, let $\Omega$ be a domain (closed) in the complex plane whose boundary $\Gamma$ is a Jordan curve, and suppose that $\Omega$ is a spectral set for $T$. Then there is a unique operator-valued measure $F$ in $\mathcal{H}$ defined on the Borel sets in $\Gamma$ which is bounded, positive and regular on $\Gamma$ and for which the integral representation (3.3) holds.

The following theorem is a version of Theorem 2.3 adjusted for the considered general case.

Theorem 3.2 Under the hypotheses of Theorem 3.1 suppose that the spectrum of $T$ is contained in the interior of the domain $\Omega$. Then the operator-valued measure $G$ representing $T$ by (3.3) is determined by

$$
G(\partial)=\int_{\partial} \Phi(\varphi(\zeta) ; \varphi(T)) d \tau,
$$

for each Borel set $\partial$ in $\partial \Omega$.

In (3.4) $\varphi$ denotes a conformal mapping of the interior of $\Omega$ on $\mathbb{D}$ extended continuously to the boundaries, $\tau$ is the normalized standard Lebesgue measure on $\partial \Omega$, and 


$$
\Phi(z ; \varphi(T))=\left(I-z \varphi(T)^{*}\right)^{-1}\left(I-\varphi(T)^{*} \varphi(T)\right)(I-\bar{z} \varphi(T))^{-1}, \quad z \in \mathbb{T} .
$$

Notice that a functional calculus can be constructed directly based on the integral representation asserted in Theorem 3.1. This functional calculus can be also deduced from that mentioned in Sect. 2 for the particular case of the unit circle. Such an approach has been developed by Sarason [11] involving spectral sets bounded by Jordan curves (see also the comments on page 54 in [14]).

Acknowledgments The research of the second author was partially supported by the Polish Ministry of Science and Higher Education, Grants; NN201546438 and 11.420.04.

Open Access This article is distributed under the terms of the Creative Commons Attribution Noncommercial License which permits any noncommercial use, distribution, and reproduction in any medium, provided the original author(s) and source are credited.

\section{References}

1. Cojuhari, A., Stochel, J.: An integral representation for general operators in Banach spaces. Int. J. Pure Appl. Math. 54(3), 451-465 (2009)

2. Diestel, J., Uhl, J.J.: Vector Measures. Mathematical Surveys; no. 15. AMS, Providence (1977)

3. Dunford, N., Schwartz, J.T.: Linear Operators, Part I: General Theory. Interscience, New York (1958)

4. Dunford, N., Schwartz, J.T.: Linear Operators, Part II: Spectral Theory. Interscience, New York (1963)

5. Dunford, N., Schwartz, J.T.: Linear Operators, Part III: Spectral Operators. Interscience, New York (1971)

6. Foiaş, C.: Some applications of spectral sets I: harmonic spectral measure. Am. Math. Soc. Transl. 61(2), 25-62 (1967)

7. Lebow, A.: On von Neumann's theory of spectral sets. J. Math. Anal. Appl. 7, 64-90 (1963)

8. Lebow, A.: Spectral radius of an absolutely continuous operator. Proc. Am. Math. Soc. 36(2), 511-514 (1977)

9. Mlak, W.: On a theorem of Lebow. Ann. Polon. Math. 35, 107-109 (1977)

10. Mlak, W.: Introduction to the Theory of Hilbert Spaces. PWN, Warsaw (1982)

11. Sarason, D.: On spectral sets having connected complement. Acta. Sci. Math. 26, 289-299 (1965)

12. Schreiber, M.: A functional calculus for general operators in Hilbert spaces. Trans. Am. Math. Soc. 87, 108-118 (1958)

13. Schreiber, M.: Absolutely continuous operators. Duke Math. J. 29, 175-190 (1962)

14. Sz-Nagy, B., Foiaş, C.: Analyse Harmoniques des Opérateurs de L'espace de Hilbert. Akad. Kiadó, Budapest (1967)

15. Riesz, F., Sz-Nagy, B.: Leçons d'Analyse Fonctionnelle. Akad. Kiadó, Budapest (1972)

16. von Neumann, J.: Eine Spektraltheorie für allgemeine Operatoren eines unitären Raumes. Math. Nachr. 4, 258-281 (1951) 